ESAIM: PROCEEDINGS, October 2007, Vol. 22, 52-66

Gabriel Caloz \& Monique Dauge, Editors

\title{
AHO SCHEMES FOR DISSIPATIVE HYPERBOLIC SYSTEMS
}

\author{
Denise Aregba-Driollet ${ }^{1}$, Maya Briani $^{2}$ And Roberto Natalini ${ }^{3}$
}

\begin{abstract}
We study finite difference schemes which approximate $2 \times 2$ linear one dimensional dissipative hyperbolic systems. We introduce suitable modifications of standard upwinding schemes to keep into account the long-time behaviour of the solutions, which consist in some schemes which are increasingly accurate for large times. This property of accuracy is required in order to get better results for large time simulations when computing perturbations of some given stable states, both in the steady state and in the diffusion limit.
\end{abstract}

Résumé. Nous présentons des schémas aux différences finies pour des systèmes hyperboliques linéaires dissipatifs $2 \times 2$ en une dimension d'espace. Afin de tenir compte du comportement asymptotique de la solution, nous modifions des schémas "upwind" classiques pour construire des approximations dont la précision augmente avec le temps. Cette technique nous permet d'améliorer sensiblement les résultats des simulations en grands temps pour les perturbations des états asymptotiquement stables, tant autour des états stationnaires que dans la limite diffusive.

\section{INTRODUCTION}

Let us consider a symmetric one-dimensional $2 \times 2$ linear system

$$
\left\{\begin{array}{l}
u_{t}+a u_{x}+b v_{x}=0, \\
v_{t}+b u_{x}+c v_{x}=-d v,
\end{array}\right.
$$

for $x \in \mathbb{R}$ and $t>0$, with the initial conditions

$$
u(x, 0)=u_{0}(x), v(x, 0)=v_{0}(x), \quad x \in \mathbb{R} .
$$

The system is hyperbolic symmetrizable, if the constants $a, b$, and $c$ are real, and dissipative under the condition $d>0$. This simple example is a useful prototype for more general nonlinear relaxation systems which arise in many different applications, see for instance [8] for examples and references. Recently, in [5] and [2], a quite complete theory for global existence and asymptotic behavior of smooth solutions for this type of systems was developed in a fully multidimensional framework. This theory, which will be briefly summarized in the one dimensional case in Section 1, needs for an extra assumption, the so-called Shizuta-Kawashima condition, see $[5,11]$, which guarantees for a sufficient coupling between the source and the transport terms. In the present

\footnotetext{
${ }^{1}$ Institut de Mathématiques de Bordeaux, UMR CNRS 5251, Université de Bordeaux 1

2 Istituto per le Applicazioni del Calcolo "Mauro Picone", Consiglio Nazionale delle Ricerche \& Università LUISS - Guido Carli di Roma

${ }^{3}$ Istituto per le Applicazioni del Calcolo "Mauro Picone", Consiglio Nazionale delle Ricerche
}

(C) EDP Sciences, SMAI 2007 
example this condition is equivalent to impose $b \neq 0$. Roughly speaking, it is possible to prove that for every small perturbation of a given stationary solution of problem (1), the corresponding solution decays in the $L^{p}$-norm to its unperturbed state as $\mathcal{O}\left(t^{-\frac{1}{2}\left(1-\frac{1}{p}\right)}\right)$, for $p \in[1, \infty]$. When the asymptotic state is given by a constant state, it is possible to compare this perturbed solution with the solution $(\hat{u}, \hat{v})$ of the Chapman-Enskog expansion, which in the present case is given by

$$
\left\{\begin{array}{l}
\hat{u}_{t}+a \hat{u}_{x}=\frac{b^{2}}{d} \hat{u}_{x x} \\
\hat{v}=-\frac{b}{d} \hat{u}_{x}
\end{array}\right.
$$

It is actually possible to prove that the difference between $u$ and $\hat{u}$ decays in the $L^{p}$-norm as $\mathcal{O}\left(t^{-\frac{1}{2}\left(2-\frac{1}{p}\right)}\right)$, for $p \in[1, \infty]$, so a factor $\frac{1}{2}$ better than the simple decay to the steady state.

In this presentation, numerical approximations related to these asymptotic results will be introduced and discussed. In [3], for a given family of stable asymptotic states for a scalar hyperbolic equations, we introduced the Asymptotic High Order (AHO) schemes, i.e.: schemes which are high-order accurate with respect to the local truncation error, when restricted to every element of this family. Here, we show that for $2 \times 2$ dissipative hyperbolic systems it is possible to introduce AHO schemes which are compatible with the behavior predicted by the qualitative analysis, both in the long-time asymptotic and Chapman-Enskog regimes. The main idea we are going to apply is to modify standard upwinding schemes to keep into account the long-time behaviour of the solutions. More details and proofs about those results will be contained in [1]. Some numerical tests will be presented here and discussed to show the better performance of our schemes with respect to the usual pointwise approximation of the source term, and even with the classical upwinding of the source proposed by Roe in 1986 [10].

\section{AnAlytical BaCKGrounds}

Let us recall some results from [2,5] about the Cauchy problem for a general hyperbolic symmetrizable one dimensional system of balance laws

$$
u_{t}+f(u)_{x}=g(u),
$$

with the initial condition

$$
u(x, 0)=u_{0}(x),
$$

where $u=\left(u_{1}, u_{2}\right) \in \Omega \subseteq \mathbb{R}^{n_{1}} \times \mathbb{R}^{n_{2}}$, with $n_{1}+n_{2}=n$. We also assume that there are $n_{1}$ conservation laws in the system, namely that we can take

$$
g(u)=\left(\begin{array}{c}
0 \\
q(u)
\end{array}\right), \text { with } q(u) \in \mathbb{R}^{n_{2}}
$$

According to the general theory of hyperbolic systems of balance laws, if the flux functions $f$ and the source term $g$ are smooth enough, it is well-known that problem (3)-(4) has a unique local smooth solution, at least for some time interval $[0, T)$ with $T>0$, if the initial data are also sufficiently smooth. In the general case, and even for nice initial data, smooth solutions may break down in finite time, due to the appearance of singularities, either discontinuities or blow-up. Despite these general considerations, sometimes dissipative mechanisms due to the source term can prevent the formation of singularities, at least for some restricted classes of initial data, as observed for many models which arise to describe physical phenomena. A typical and well-known example is given by the compressible Euler equations with damping, see [6,9] in one space dimension and [12] in three space dimensions.

In [5], it was proposed a general framework of sufficient conditions which guarantee the global existence in time of smooth solutions. Actually, for the systems which are endowed with a strictly convex entropy function 
$\mathcal{E}=\mathcal{E}(u)$, a first natural assumption is the entropy dissipation condition, see [4], namely for every $u, \bar{u} \in \Omega$, with $g(\bar{u})=0$,

$$
\left(\mathcal{E}^{\prime}(u)-\mathcal{E}^{\prime}(\bar{u})\right) \cdot g(u) \leq 0
$$

Unfortunately, it is easy to see that this condition is too weak to prevent the formation of singularities. A quite natural supplementary condition can be imposed to entropy dissipative systems, following the classical approach by Shizuta and Kawashima [7,11], which in the present case reads

$$
\operatorname{Ker} D g(\bar{u}) \cap\{\text { eigenspaces of } D f(\bar{u})\}=\{0\} \text {, }
$$

for every $\bar{u} \in \Omega$, with $g(\bar{u})=0$. It is possible to prove that this condition, which is satisfied in many interesting examples, is also sufficient to establish a result of global existence for small perturbations of equilibrium constant states, see [5].

In [2], some results have been obtained about the asymptotic behavior in time of the global solutions, then always assuming the existence of a strictly convex dissipative entropy and condition (6). One of the main point of this theory is the existence of a canonical projections on two different components: the conservative part and the dissipative part. The first one, which loosely speaking corresponds to the conservative part of equations in (3), decays in time like the heat kernel. On the other side, the dissipative part is strongly influenced by the dissipation and decays at a rate $t^{-\frac{1}{2}}$ faster of the conservative one. Actually the main result of decay, in the general nonlinear case, can be stated as follows. Let $u_{c}$ be the conservative part and $u_{d}$ be the dissipative part of the solution. For $s>0$, let $E_{s}=\max \left\{\|u(0)\|_{L^{1}},\|u(0)\|_{H^{s}}\right\}$.

Theorem 1.1. Let $u(t)=\left(u_{c}(t), u_{d}(t)\right)$ be a smooth global solution to problem $(3)-(4)$, for a system endwed with a strictly convex dissipative entropy and under condition (6). Assume $E_{3}$ small enough and let $p \in[1, \infty]$. The following decay estimate holds

$$
\left\|D^{\beta} u(t)\right\|_{L^{p}} \leq C \min \left\{1, t^{-\frac{1}{2}\left(1-\frac{1}{p}\right)-|\beta| / 2}\right\} E_{|\beta|+2}
$$

with $C=C\left(E_{|\beta|+\sigma}\right)$, for $\sigma$ large enough. For the dissipative part, we have a more precise estimate:

$$
\left\|D^{\beta} u_{d}(t)\right\|_{L^{p}} \leq C \min \left\{1, t^{-\frac{1}{2}\left(1-\frac{1}{p}\right)-1 / 2-|\beta| / 2}\right\} E_{|\beta|+2}
$$

Another interesting result concerns the convergence to the Chapman-Enskog expansion of problem (3), which for the general one dimensional case is given by the following nonlinear equation

$$
w_{t}+\left(D f(0)_{c c} w+\tilde{A}(w, w)\right)_{x}+D f(0)_{c d}\left(D_{u_{d}} q(0)\right)^{-1} D f(0)_{d c} w_{x x}=0
$$

where the bilinear forme $A(w, w)$ is defined in terms of the derivatives of $D f(0)$ and $D_{u_{d}} q(0)$, see [2] for more details. In this case it is possible to prove the following result.

Theorem 1.2. Let $u_{p}$ be the solution of problem (9), with a suitable initial condition. Under the assumptions of Theorem 1.1, we have the following more accurate decay estimate

$$
\left\|D^{\beta}\left(u_{c}(t)-u_{p}(t)\right)\right\|_{L^{p}} \leq C \min \left\{1, t^{-\frac{1}{2}\left(1-\frac{1}{p}\right)-|\beta| / 2-1 / 2}\right\} E_{|\beta|+2}
$$

with $C=C\left(E_{|\beta|+\sigma}\right)$, for $\sigma$ large enough.

Given every $2 \times 2$ entropy dissipative hyperbolic system in one space dimension, it is possible to prove that its linearized version can be reduced to the form (1) by a simple change of variables, see again [2]. Moreover, for system (1), the condition (6) it is equivalent to say that $b \neq 0$. Let us remark that, thanks to the symmetry of the coefficients of the transport terms, the condition of entropy dissipation is always verified. It also possible to 
see that, for this simple example, the condition of entropy dissipation with (6), is equivalent to the celebrated sub-characteristic condition by Whitham [13]:

$$
\lambda_{1}<a<\lambda_{2}
$$

where $\lambda_{1,2}=\left(a+c \pm \sqrt{(a-c)^{2}+4 b^{2}}\right) / 2$ are the (real) eigenvalues associated to the matrix

$$
A=\left(\begin{array}{ll}
a & b \\
b & c
\end{array}\right)
$$

It is easy to see that the Chapman-Enskog expansion (9) is now given by (2) and that both Theorem 1.1 and 1.2 hold true, using for the conservative part $u_{c}=u$ and for the dissipative part $u_{d}=v$. In this specific case, some stronger estimates are available, which is what we are going to review now. First of all we have a contraction of the solution in the $L^{2}$-norm.

Proposition 1.3 ( $L^{2}$-stability). Under the assumptions given above

$$
\sum_{i=1,2} \int_{\Omega}\left|u_{i}(x, t)\right|^{2} \leq \sum_{i=1,2} \int_{\Omega}\left|u_{i}(x, 0)\right|^{2} .
$$

To obtain the estimates for the $L^{1}, L^{\infty}$ and BV norms, we need to diagonalize the system. This operation can be performed by diagonalizing the matrix $A$, namely

$$
A=R \Lambda R^{-1}
$$

where $\Lambda=\operatorname{diag}\left(\lambda_{1}, \lambda_{2}\right)$, and $R=\left(r^{(1)}, r^{(2)}\right)$ is the column matrix of right eingeinvectors, i.e. $A r^{(i)}=\lambda_{i} r^{(i)}$, $i=1,2$. Introducing the notation

$$
\mathrm{w}=R^{-1} \mathrm{u}
$$

problem (1) becomes,

$$
\left\{\begin{array}{l}
\partial_{t} \mathrm{w}+\Lambda \partial_{x} \mathrm{w}=\tilde{B} \mathrm{w}, \\
\mathrm{w}(x, 0)=\mathrm{w}^{0}(x)=R^{-1} \mathrm{u}^{0}(x),
\end{array}\right.
$$

where $\tilde{B}=R^{-1} B R$.

Now it is possible to see that, if we choose the matrix of the diagonalization of the system (1) as

$$
R=\left(\begin{array}{cc}
1 & 1 \\
-\frac{\left(a-\lambda_{1}\right)}{b} & \frac{\left(\lambda_{2}-a\right)}{b}
\end{array}\right),
$$

then the corresponding source term is given by

$$
\tilde{B}=\frac{d}{\lambda_{2}-\lambda_{1}}\left(\begin{array}{cc}
-\left(a-\lambda_{1}\right) & \left(\lambda_{2}-a\right) \\
\left(a-\lambda_{1}\right) & -\left(\lambda_{2}-a\right)
\end{array}\right) \text {. }
$$

Now we can state the main result about monotonicity and $L^{1}$-contraction properties of system (1). Let us set

$$
L=L(\lambda)=\max _{i=1,2}\left|\lambda_{i}\right|
$$

For any given vectors $C, D \in \mathbb{R}^{2}$, set $C \leq D$ if $c_{i} \leq d_{i}(i=1,2)$; if $C \leq D$, let us denote the corresponding interval by $[C, D]$. Also, for each interval $I=(\alpha, \beta) \subseteq \mathbb{R}$, we set $\tau=(\beta-\alpha) / 2 L$. 
Proposition 1.4. Let $\mathrm{w}$ and $\tilde{\mathrm{w}}$ be two weak solutions of the Cauchy problem (13) in $\mathbb{R} \times(0, T)(T>0)$, for the initial data $\mathrm{w}^{0}$ and $\tilde{\mathrm{w}}^{0}$ respectively. Let $[C, D] \subseteq \mathbb{R}^{2}$ be an interval with non empty interior such that $\mathrm{w}$, $\tilde{\mathrm{w}} \in[C, D]$. If the matrix $\tilde{B}$ is given by (15), then for each interval $(\alpha, \beta) \subseteq \mathbb{R}$ and for all $t \in(0, \min (\tau, T))$, we have

$$
\sum_{i=1,2} \int_{\alpha+t L}^{\beta-t L}\left[w_{i}-\tilde{w}_{i}\right]_{+} d x \leq \sum_{i=1,2} \int_{\alpha}^{\beta}\left[w_{i}^{0}-\tilde{w}_{i}^{0}\right]_{+} d x
$$

Clearly, from Proposition 1.4, we can easily deduce, by standard arguments, not only the comparison principle and the $L^{1}$-estimates, but also the a priori BV-estimates for the solutions to problem (1).

\section{Numerical approximation}

Here we present a general finite difference approximation for system (1) and we show some convergence properties.

\subsection{Construction of the schemes}

To deal with the approximation of the differential part following the direction of the characteristic velocities, we study first the methods for the system in diagonal form (13) obtained by the choice (14). We denote $\mathrm{w}=\left(w^{1}, w^{2}\right)$ the exact solution.

We denote $h$ the uniform mesh-length and $x_{l}=l h$ the spatial grid points for all $l \in \mathbb{Z}$. The time levels $t_{n}$, with $t_{0}=0$, are also spaced uniformly with mesh-length $\Delta t=t_{n+1}-t_{n}$ for $n \in \mathbb{N}$. We denote by $\delta$ the CFL ratio $\delta=\Delta t / h$, which is taken constant through the paper.

We consider the Cauchy problem (13). The initial data $\mathrm{w}^{0}$ is supposed to be smooth and is approximated by its node values. The approximate solution $W_{l}^{n}=\left(w_{l, n}^{1}, w_{l, n}^{2}\right)^{T}$ is given by

$$
\begin{aligned}
& \frac{W_{l}^{n+1}-W_{l}^{n}}{\Delta t}+\frac{\Lambda}{2 h}\left(W_{l+1}^{n}-W_{l-1}^{n}\right)-\frac{\tilde{Q}}{2 h}\left(W_{l+1}^{n}-2 W_{l}^{n}+W_{l-1}^{n}\right) \\
& =\tilde{\mathcal{B}}_{-1} W_{l-1}^{n}+\tilde{\mathcal{B}}_{0} W_{l}^{n}+\tilde{\mathcal{B}}_{1} W_{l+1}^{n}, \quad l \in \mathbb{Z}, n \in \mathbb{N} \\
& W_{l}^{0}=\mathrm{w}^{0}\left(x_{l}\right), \quad l \in \mathbb{Z},
\end{aligned}
$$

where $\tilde{Q}=\operatorname{diag}\left(\tilde{q}_{1}, \tilde{q}_{2}\right)$ is the diagonal matrix of the artificial diffusion terms $\tilde{q}_{i} \geq 0(i=1,2)$, and $\tilde{\mathcal{B}}_{-1}=$ $\left(\tilde{\beta}_{i j}^{-1}\right)_{i, j=1,2}, \tilde{\mathcal{B}}_{0}=\left(\tilde{\beta}_{i j}^{0}\right)_{i, j=1,2}$ and $\tilde{\mathcal{B}}_{1}=\left(\tilde{\beta}_{i j}^{1}\right)_{i, j=1,2}$ are $2 \times 2$ constant matrices that define the source approximation. Those matrices may depend on $h$.

We denote

$$
W_{\Delta}^{n}(x)=W_{l}^{n} \quad \text { if } x \in\left[x_{l}, x_{l+1}[\right.
$$

and

$$
W_{\Delta}(x, t)=W_{\Delta}^{n}(x) \quad \text { if } t \in\left[t_{n}, t_{n+1}[.\right.
$$

The scheme (17) can be seen as a linear function

$$
W_{\Delta}^{n+1}=\mathbf{S}_{\Delta t}\left(W_{\Delta}^{n}\right)
$$

More precisely we have

$$
w_{l, n+1}^{i}=S^{i}\left(W_{l-1}^{n}, W_{l}^{n}, W_{l+1}^{n}\right), \quad i=1,2, \quad l \in \mathbb{Z}
$$


with

$$
\begin{aligned}
& S^{i}\left(W_{l-1}^{n}, W_{l}^{n}, W_{l+1}^{n}\right)=\left(\alpha_{-1}^{i} w_{l-1, n}^{i}+\alpha_{0}^{i} w_{l, n}^{i}+\alpha_{1}^{i} w_{l+1, n}^{i}\right) \\
& +\Delta t \sum_{j \in \Gamma}\left(\tilde{\beta}_{i j}^{-1} w_{l-1, n}^{j}+\tilde{\beta}_{i j}^{0} w_{l, n}^{j}+\tilde{\beta}_{i j}^{1} w_{l+1, n}^{j}\right)
\end{aligned}
$$

where, for all $i=1,2$,

$$
\alpha_{-1}^{i}=\frac{\delta}{2} \lambda_{i}+\frac{\delta}{2} \tilde{q}_{i}, \quad \alpha_{0}^{i}=1-\delta \tilde{q}_{i}, \quad \alpha_{1}^{i}=-\frac{\delta}{2} \lambda_{i}+\frac{\delta}{2} \tilde{q}_{i} .
$$

For any function $\psi$ defined on $\mathbb{R}$ with values in $\mathbb{R}^{2}$, we denote

$$
\bar{\psi}(x)=(\psi(x-h), \psi(x), \psi(x+h)) .
$$

The function $\mathbf{S}_{\Delta t}$ is then defined by

$$
\mathbf{S}_{\Delta t}(\psi)(x)=\left(S^{1}(\bar{\psi}(x)), S^{2}(\bar{\psi}(x))\right) .
$$

\subsection{Convergence for smooth data}

We assume that the scheme satisfies the two following properties:

(1) Consistency

The scheme (17) is consistent with problem (13), i.e

$$
\tilde{\mathcal{B}}_{-1}+\tilde{\mathcal{B}}_{0}+\tilde{\mathcal{B}}_{1}=\tilde{B}+h \tilde{C},
$$

where $\tilde{C}=\left(\tilde{c}_{i j}\right)_{i, j=1,2}$ is a $2 \times 2$ constant matrix not depending on $h$ and $\Delta t$.

(2) Monotonicity

For all $i=1,2$, the operators $S^{i}$, defined in (19), are monotone nondecreasing in all their components, i.e.

$$
\begin{aligned}
& \tilde{\beta}_{i j}^{-1}, \tilde{\beta}_{i j}^{0}, \tilde{\beta}_{i j}^{1} \geq 0 \quad \forall i, j \in \Gamma, i \neq j, \\
& A_{i, 0}=1-\delta \tilde{q}_{i}+\Delta t \tilde{\beta}_{i i}^{0} \geq 0, \quad \forall i=1,2, \\
& A_{i,-1}=\frac{\lambda_{i}}{2 h}+\frac{\tilde{q}_{i}}{2 h}+\tilde{\beta}_{i i}^{-1} \geq 0, \quad A_{i, 1}=-\frac{\lambda_{i}}{2 h}+\frac{\tilde{q}_{i}}{2 h}+\tilde{\beta}_{i i}^{1} \geq 0, \quad \forall i=1,2 .
\end{aligned}
$$

For $t \geq 0$ and $x \in \mathbb{R}$, by using the notation (22) for $\mathrm{w}$, the local truncation error $T(x, t)$ is defined by

$$
T(x, t)=\frac{\mathrm{w}(x, t+\Delta t)-\mathbf{S}_{\Delta t} \mathrm{w}(., t)(x)}{\Delta t}
$$

which can also be written

$$
T^{i}(x, t)=\frac{w^{i}(x, t+\Delta t)-S^{i}(\overline{\mathrm{w}}(x))}{\Delta t}, \quad i \in \Gamma .
$$

It is obtained straightforwardly by Taylor expansions:

$$
\begin{aligned}
T^{i}(x, t)= & \frac{\Delta t}{2} \partial_{t t} w^{i}(x, t+\theta \Delta t)-h \frac{\tilde{q}_{i}}{2} \partial_{x x} w^{i}(x, t)-h \sum_{j \in \Gamma} \tilde{c}_{i j} w^{j}(x, t) \\
& -h \sum_{j \in \Gamma}\left(\tilde{\beta}_{i j}^{1}-\tilde{\beta}_{i j}^{-1}\right) \partial_{x} w^{i}(x, t)+O\left(h^{2}\right) .
\end{aligned}
$$


In this formula, $\theta \in[0,1]$ and the last term depends on the spatial derivatives of $\mathrm{w}$, up to third order.

In view of proving convergence, we need to define the discrete truncation error, which is

$$
T_{\Delta}(x, t)=T\left(x_{l}, t\right) \quad \text { for } \quad x \in\left[x_{l}, x_{l+1}[.\right.
$$

The scheme is first order in the following sense:

Proposition 2.1 (Accuracy for smooth data). Consider a smooth initial data $\mathrm{w}^{0}$ such that

$$
\frac{d^{k} \mathrm{w}^{0}}{d x^{k}} \in L^{1}(\mathbb{R}) \cap L^{\infty}(\mathbb{R}) \quad \text { for } 0 \leq k \leq 4
$$

Then the Cauchy problem (13) has a unique solution $\mathrm{w} \in C^{0}\left(\mathbb{R}^{+}, L^{1}(\mathbb{R})\right)$ and for all $k \leq 4, \partial_{x}^{k} \mathrm{w} \in C^{0}\left(\mathbb{R}^{+}, L^{1}(\mathbb{R})\right) \cap$ $L^{\infty}\left(\left[0,+\infty[\times \mathbb{R})\right.\right.$. Moreover, the discrete local truncation error $T_{\Delta}(x, t)$ for the scheme (17) satisfies

$$
\sum_{i \in \Gamma}\left[\left\|T_{\Delta}^{i}(., t)\right\|_{1}+\left\|T_{\Delta}^{i}(., t)\right\|_{\infty}\right] \leq C(\Delta t+h) .
$$

Here

$$
C \leq C_{1} \sup _{k \leq 4} \sup _{t \in[0,+\infty[}\left(\left\|\partial_{x}^{k} \mathrm{w}(., t)\right\|_{1}+\left\|\partial_{x}^{k} \mathrm{w}(., t)\right\|_{\infty}\right)
$$

and $C_{1}$ is a uniform constant.

We show now that the scheme is stable.

Proposition 2.2 ( $L^{1}$ stability). For the matrix $\tilde{C}$ defined in (24), we introduce the quantity

$$
\tilde{\gamma}=\left[\sup _{i \in \Gamma}\left\{\tilde{c}_{i i}+\sum_{j \neq i} \tilde{c}_{j i}\right\}\right]_{+} .
$$

Under the monotonicity assumptions (25-27), the scheme (17) is $L^{1}$-stable: for all $W_{\Delta}^{0} \in L^{1}(\mathbb{R})$ it holds

$$
\left\|W_{\Delta}^{n+1}\right\|_{1} \leq(1+\tilde{\gamma} h \Delta t)\left\|W_{\Delta}^{n}\right\|_{1}, \quad \forall n \geq 0 .
$$

Remark 2.3. As the scheme is linear, this property implies that the scheme is $L^{1}$ stable and TVB.

We have also a uniform bound for the scheme:

Proposition 2.4 ( $L^{\infty}$ stability). We make the same assumptions as in proposition (2.2). The scheme (17) is $L^{\infty}$-stable: for all $W_{\Delta}^{0} \in L^{\infty}(\mathbb{R})$ it holds

$$
\left\|W_{\Delta}^{n}\right\|_{\infty} \leq(1+\tilde{\gamma} h \Delta t)\left\|W_{\Delta}^{n-1}\right\|_{\infty} \leq e^{\tilde{\gamma} h t_{n}}\left\|W_{\Delta}^{0}\right\|_{\infty}, \quad \forall n \geq 1 .
$$

We have proved consistency and stability, so we have convergence.

Theorem 2.5. Let $\mathrm{w}^{0}$ be a smooth initial data satisfying (29). We suppose that the monotonicity assumptions (25-27) are verified. Then for all $T>0$ the scheme (17) converges in $L^{\infty}\left([0, T], L^{1}(\mathbb{R}) \cap L^{\infty}(\mathbb{R})\right)$ towards the solution of the Cauchy problem (13). More precisely, the following estimate holds:

$$
\sup _{t \in[0, T]}\left(\left\|W_{\Delta}(., t)-\mathrm{w}(., t)\right\|_{1}+\left\|W_{\Delta}(., t)-\mathrm{w}(., t)\right\|_{\infty}\right) \leq(2 T+1) C(\Delta t+h)
$$

where $C$ is defined in (31). 


\section{Asymptotic High-Order Schemes}

In this section, we study the discretization of the source term, defined by coefficients $\tilde{\mathcal{B}}_{-1,0,1}$ introduced in (13), to present some schemes which are increasingly accurate for large times, with respect to the asymptotic behavior of solutions. This property of accuracy is required in order to get better results for large time simulations when computing perturbations of non constant stable states. Actually, given a stable solution $z$ (or a family of stable solutions), we say that a scheme is Asymptotic High-Order (AHO) with respect to this stable state if the scheme is high-order accurate when restricted to this solution, see [3].

\subsection{The construction of an AHO4 scheme for stationary solutions}

Here, we consider the case of a perturbation of a generic stationary solution. Let us first develop the formula (28) for a smooth solution w:

$$
\begin{aligned}
& T(x, t)=\frac{\Lambda}{2 h}\left(\frac{h^{3}}{3} \partial_{x}^{3} \mathrm{w}(x, t)+O\left(h^{5}\right)\right) \\
& -\frac{\tilde{Q}}{2 h}\left(h^{2} \partial_{x}^{2} \mathrm{w}(x, t)+\frac{h^{4}}{12} \partial_{x}^{4} \mathrm{w}(x, t)+O\left(h^{6}\right)\right) \\
& -h \tilde{C} \mathrm{w}(x, t)-h\left(\tilde{\mathcal{B}}_{1}-\tilde{\mathcal{B}}_{-1}\right) \partial_{x} \mathrm{w}(x, t)-\frac{h^{2}}{2}\left(\tilde{\mathcal{B}}_{1}+\tilde{\mathcal{B}}_{-1}\right) \partial_{x}^{2} \mathrm{w}(x, t) \\
& -\frac{h^{3}}{6}\left(\tilde{\mathcal{B}}_{1}-\tilde{\mathcal{B}}_{-1}\right) \partial_{x}^{3} \mathrm{w}(x, t)+O\left(h^{4}\right)+\frac{\Delta t}{2} \partial_{t}^{2} \mathrm{w}(x, t+\theta \Delta t) .
\end{aligned}
$$

The terms $O\left(h^{k}\right)$ involve only spatial derivatives of w. Let us now consider the stationary solution z, namely the solution of problem

$$
\Lambda \partial_{x} \mathrm{z}=\tilde{B} \mathrm{z}
$$

For smooth data and since $\operatorname{det} A=\operatorname{det} \Lambda \neq 0$, we get

$$
\partial_{x}^{k} \mathrm{z}=\left(\Lambda^{-1} \tilde{B}\right)^{k} \mathrm{z}
$$

We compute the local truncation error (35) on this particular solution. It does not depend on $t$ anymore and we denote it by $T_{\text {stat }}(x)$ :

$$
\begin{aligned}
T_{\text {stat }}(x)= & -\frac{h}{2}\left(\tilde{Q}\left(\Lambda^{-1} \tilde{B}\right)^{2}+2 \tilde{C}+2\left(\tilde{\mathcal{B}}_{1}-\tilde{\mathcal{B}}_{-1}\right)\left(\Lambda^{-1} \tilde{B}\right)\right) \mathrm{z}(x) \\
& -\frac{h^{2}}{6}\left(-\tilde{B}+3\left(\tilde{\mathcal{B}}_{1}+\tilde{\mathcal{B}}_{-1}\right)\right)\left(\Lambda^{-1} \tilde{B}\right)^{2} \mathrm{z}(x) \\
& -\frac{h^{3}}{24}\left(\tilde{Q}\left(\Lambda^{-1} \tilde{B}\right)+4\left(\tilde{\mathcal{B}}_{1}-\tilde{\mathcal{B}}_{-1}\right)\right)\left(\Lambda^{-1} \tilde{B}\right)^{3} \mathrm{z}(x)+O\left(h^{4}\right) .
\end{aligned}
$$

Therefore, we get an AHO4 scheme as soon as all terms between round brackets vanish. The AHO4-coefficients $\tilde{\mathcal{B}}_{-1}, \tilde{\mathcal{B}}_{0}, \tilde{\mathcal{B}}_{1}$ are then univoquely defined by

$$
\left\{\begin{array}{l}
\tilde{\mathcal{B}}_{-1}=\tilde{B} / 6+\tilde{Q} \Lambda^{-1} \tilde{B} / 8 \\
\tilde{\mathcal{B}}_{0}=2 \tilde{B} / 3-h \tilde{Q}\left(\Lambda^{-1} \tilde{B}\right)^{2} / 4 \\
\tilde{\mathcal{B}}_{1}=\tilde{B} / 6-\tilde{Q} \Lambda^{-1} \tilde{B} / 8
\end{array}\right.
$$


or equivalently

$$
\begin{gathered}
\tilde{\mathcal{B}}_{a h o}^{-1}=\frac{1}{8}\left[\begin{array}{ll}
\tilde{b}_{11}\left(4 / 3+\tilde{q}_{1} / \lambda_{1}\right) & \tilde{b}_{12}\left(4 / 3+\tilde{q}_{1} / \lambda_{1}\right) \\
\tilde{b}_{21}\left(4 / 3+\tilde{q}_{2} / \lambda_{2}\right) & \tilde{b}_{22}\left(4 / 3+\tilde{q}_{2} / \lambda_{2}\right)
\end{array}\right], \\
\tilde{\mathcal{B}}_{a h o}^{0}=\frac{2}{3}\left[\begin{array}{ll}
\tilde{b}_{11} & \tilde{b}_{12} \\
\tilde{b}_{21} & \tilde{b}_{22}
\end{array}\right]+\frac{a d h}{4 \lambda_{1}^{2} \lambda_{2}^{2}}\left[\begin{array}{cc}
\lambda_{2} \tilde{q}_{1} \tilde{b}_{11} & \lambda_{2} \tilde{q}_{1} \tilde{b}_{12} \\
\lambda_{1} \tilde{q}_{2} \tilde{b}_{21} & \lambda_{1} \tilde{q}_{2} \tilde{b}_{22}
\end{array}\right], \\
\tilde{\mathcal{B}}_{a h o}^{1}=\frac{1}{8}\left[\begin{array}{ll}
\tilde{b}_{11}\left(4 / 3-\tilde{q}_{1} / \lambda_{1}\right) & \tilde{b}_{12}\left(4 / 3-\tilde{q}_{1} / \lambda_{1}\right) \\
\tilde{b}_{21}\left(4 / 3-\tilde{q}_{2} / \lambda_{2}\right) & \tilde{b}_{22}\left(4 / 3-\tilde{q}_{2} / \lambda_{2}\right)
\end{array}\right] .
\end{gathered}
$$

The scheme is of fourth order for long times, as expressed in the following proposition:

Proposition 3.1 (Asymptotic high order). Let $\mathrm{z}$ be a smooth stationary solution of system (13). Let $\mathrm{w}_{0}$ be a small and smooth perturbation of $\mathrm{z}$.

Then, for any compact set $K \subseteq \mathbb{R}$, there exists a constant $C_{4}(K)$ such that the local truncation error $T(x, t)$ of the scheme (13)-(39) satisfies:

$$
\|T(., t)\|_{L^{\infty}(K)} \leq C_{4}(K) \min \left(\Delta t+h, h^{4}+\frac{1}{\Delta t \sqrt{t}}\right) .
$$

Remark 3.2. Let us remark that assuming on $\tilde{\mathcal{B}}_{1}, \tilde{\mathcal{B}}_{-1}$ that

$$
\tilde{Q}\left(\Lambda^{-1} \tilde{B}\right)^{2}+2 \tilde{C}+2\left(\tilde{\mathcal{B}}_{1}-\tilde{\mathcal{B}}_{-1}\right)\left(\Lambda^{-1} \tilde{B}\right)=0,
$$

we get a class of AHO2 schemes, while for (41) and

$$
-\tilde{B}+3\left(\tilde{\mathcal{B}}_{1}+\tilde{\mathcal{B}}_{-1}\right)=0,
$$

the schemes are asymptotically of third-order.

Proposition 3.3 (Monotonicity). The scheme (13), coupled with (39) satisfies the monotonicity requirements (25 - 27) under the following assumptions:

$$
\begin{gathered}
h<\min _{1 \leq i \leq 2} \frac{3\left|\lambda_{i}\right|}{\left|\tilde{b}_{i i}\right|}, \\
h \leq\left|\frac{2 \lambda_{1} \lambda_{2}}{a d}\right| \quad \text { if } \min \left(a \lambda_{1}, a \lambda_{2}\right)<0, \\
1+h \frac{\left|\tilde{b}_{i i}\right|}{3\left(4\left|\lambda_{i}\right|+h\left|\tilde{b}_{i i}\right|\right)} \leq \frac{\tilde{q}_{i}}{\left|\lambda_{i}\right|} \leq \frac{4}{3}, \quad i=1,2,
\end{gathered}
$$

and

$$
\frac{\Delta t}{h} \leq \min _{1 \leq i \leq 2}\left(\tilde{q}_{i}+h\left|\tilde{b}_{i i}\right|\left(\frac{a d h \tilde{q}_{i}}{4 \lambda_{1} \lambda_{2} \lambda_{i}}+\frac{2}{3}\right)\right)^{-1} .
$$

Moreover these conditions are not empty.

In the following we shall compare the AHO4 approximation (39) to these two schemes,

Example 3.1 (source term pointwise approximation (AHO1-UP)).

$$
\tilde{B}_{u p}^{-1}=\left[\begin{array}{ll}
0 & 0 \\
0 & 0
\end{array}\right], \quad \tilde{B}_{u p}^{0}=\left[\begin{array}{cc}
\tilde{b}_{11} & \tilde{b}_{12} \\
\tilde{b}_{21} & \tilde{b}_{22}
\end{array}\right], \quad \tilde{B}_{u p}^{1}=\left[\begin{array}{cc}
0 & 0 \\
0 & 0
\end{array}\right] .
$$


Example 3.2 (upwinding of the source term (AHO2-ROE), [10]).

$$
\tilde{B}_{\text {roe }}^{-1}=\left[\begin{array}{cc}
H\left(\lambda_{1}\right) \tilde{b}_{11} & H\left(\lambda_{1}\right) \tilde{b}_{12} \\
H\left(\lambda_{2}\right) \tilde{b}_{21} & H\left(\lambda_{2}\right) \tilde{b}_{22}
\end{array}\right], \quad \tilde{B}_{\text {roe }}^{0}=\frac{1}{2}\left[\begin{array}{ll}
\tilde{b}_{11} & \tilde{b}_{12} \\
\tilde{b}_{21} & \tilde{b}_{22}
\end{array}\right], \quad \tilde{B}_{\text {roe }}^{1}=\left[\begin{array}{cc}
\left(1-H\left(\lambda_{1}\right)\right) \tilde{b}_{11} & \left(1-H\left(\lambda_{1}\right)\right) \tilde{b}_{12} \\
\left(1-H\left(\lambda_{2}\right)\right) \tilde{b}_{21} & \left(1-H\left(\lambda_{2}\right)\right) \tilde{b}_{22}
\end{array}\right],
$$

where $H(\cdot)$ is the Heaviside function.

\subsection{Consistency with the diffusion limit}

In this section we shall consider the $2 \times 2$ linear system (1), to study a numerical scheme which is high-order accurate with respect to the parabolic asymptotic problem.

We shall consider for simplicity only the case $c=-a$ looking at the adimensional problem

$$
\left\{\begin{array}{l}
u_{t}+\alpha u_{x}+v_{x}=0 \\
v_{t}+u_{x}-\alpha v_{x}=-\beta v
\end{array}\right.
$$

where $\alpha=a / b$ and $\beta=t^{*} d$, for a given time scale $t^{*}$. The Chapman-Enskog limit of system (49) gives

$$
\left\{\begin{array}{l}
\hat{u}_{t}+\alpha \hat{u}_{x}=\frac{1}{\beta} \hat{u}_{x x} \\
\hat{v}=-\frac{1}{\beta} \hat{u}_{x}
\end{array}\right.
$$

Let us consider the following finite difference approximation for problem (49),

$$
\begin{aligned}
& \frac{U_{j}^{n+1}-U_{j}^{n}}{\Delta t}+\frac{A}{2 h}\left(U_{j+1}^{n}-U_{j-1}^{n}\right)-\frac{R \tilde{Q} R^{-1}}{2 h}\left(U_{j+1}^{n}-2 U_{j}^{n}+U_{j-1}^{n}\right) \\
& =R \tilde{\mathcal{B}}_{-1} R^{-1} U_{j-1}^{n}+R \tilde{\mathcal{B}}_{0} R^{-1} U_{j}^{n}+R \tilde{\mathcal{B}}_{1} R^{-1} U_{j+1}^{n},
\end{aligned}
$$

where $\lambda=\sqrt{1+\alpha^{2}}$ and

$$
R=\left(\begin{array}{cc}
1 & 1 \\
-(\alpha+\lambda) & \lambda-\alpha
\end{array}\right), \quad R^{-1}=\frac{1}{2 \lambda}\left(\begin{array}{cc}
\lambda-\alpha & -1 \\
\alpha+\lambda & 1
\end{array}\right)
$$

We fix $\tilde{Q}$ as

then

$$
\tilde{Q}=\left(\begin{array}{cc}
\lambda & 0 \\
0 & \lambda
\end{array}\right)
$$

$$
Q=R \tilde{Q} R^{-1}=\tilde{Q} .
$$

By Taylor expansion, the numerical approximation (51) is consistent with system

$$
U_{t}+A U_{x}-B U=h\left[\frac{Q}{2} U_{x x}+\left(B_{1}-B_{-1}\right) U_{x}+C U\right]+O\left(h^{2}+\Delta t\right) .
$$

Going into details, for differents schemes, the Chapman-Enskog limit of system (52) gives,

- source term pointwise approximation (AHO1-UP) given by (47)

$$
B_{1}-B_{-1}=0, \quad C=0,
$$




$$
\left\{\begin{array}{l}
\hat{u}_{t}+\alpha \hat{u}_{x}=\left(\frac{1}{\beta}+\frac{h \lambda}{2}\right) \hat{u}_{x x} \\
\hat{v}=-\frac{1}{\beta} \hat{u}_{x}
\end{array}\right.
$$

- upwinding of the source term (AHO2-ROE) given by (48)

$$
\begin{aligned}
& B_{1}-B_{-1}=\frac{\beta}{2 \lambda}\left(\begin{array}{cc}
0 & 1 \\
0 & -\alpha
\end{array}\right), \quad C=0, \\
& \left\{\begin{array}{l}
\hat{u}_{t}+\alpha \hat{u}_{x}=\left(\frac{1}{\beta}+h \frac{\lambda}{2}-h \frac{1}{2 \lambda}\right) \hat{u}_{x x}, \\
\hat{v}=-\frac{1}{\beta} \hat{u}_{x}
\end{array}\right.
\end{aligned}
$$

- Asymptotic High Order (AHO4) given by (39)

$$
\begin{gathered}
B_{1}-B_{-1}=\frac{\beta}{4 \lambda}\left(\begin{array}{cc}
0 & 1 \\
0 & -\alpha
\end{array}\right), \quad C=\frac{\alpha \beta^{2}}{4 \lambda^{3}}\left(\begin{array}{cc}
0 & 1 \\
0 & -\alpha
\end{array}\right), \\
\left\{\begin{array}{l}
\hat{u}_{t}+\alpha\left(1+h \frac{\alpha \beta}{\left(4 \lambda^{3}+h \alpha^{2} \beta\right)}\right) \hat{u}_{x}=\left[\frac{4 \lambda^{3}}{\beta\left(4 \lambda^{3}+h \alpha^{2} \beta\right)}+h \frac{\lambda}{2}-h \frac{\lambda^{2}}{\left(4 \lambda^{3}+h \alpha^{2} \beta\right)}\right] \hat{u}_{x x} \\
\hat{v}=-\frac{4 \lambda^{3}}{\beta\left(4 \lambda^{3}+h \alpha^{2} \beta\right)} \hat{u}_{x}
\end{array}\right.
\end{gathered}
$$

Notice that, for all schemes the consistency with the parabolic system (50) is of order $O(h)$. In all cases, the diffusive term has a perturbation of order $O(h)$. In particular, the pointwise approximation is the most diffusive one. Moreover, we observe that the AHO4 approximation has a perturbation of order $O(h)$ also on the drift term.

We then look for a scheme at least of order $O\left(h^{2}\right)$ with respect to the parabolic problem (50).

\subsubsection{AHO on the parabolic profile (AHOp)}

Let us consider system (52) with $Q=\tilde{Q}=\operatorname{diag}(q, q)$,

$$
\left\{\begin{array}{l}
u_{t}+\alpha u_{x}+v_{x}=h\left[\frac{q}{2} u_{x x}+\left(\beta_{11}^{1}-\beta_{11}^{-1}\right) u_{x}+\left(\beta_{12}^{1}-\beta_{12}^{-1}\right) v_{x}+c_{11} u+c_{12} v\right]+O\left(h^{2}+\Delta t\right), \\
v_{t}+u_{x}-\alpha v_{x}+\beta v=h\left[\frac{q}{2} v_{x x}+\left(\beta_{21}^{1}-\beta_{21}^{-1}\right) u_{x}+\left(\beta_{22}^{1}-\beta_{22}^{-1}\right) v_{x}+c_{21} u+c_{22} v\right]+O\left(h^{2}+\Delta t\right) .
\end{array}\right.
$$

Assuming enough regularity on the data, the decay properties of the solution allow to consider, when $t$ goes to $+\infty$, the following reduced system,

$$
\left\{\begin{array}{l}
u_{t}+\alpha u_{x}+v_{x}=h\left[\frac{q}{2} u_{x x}+\left(\beta_{11}^{1}-\beta_{11}^{-1}\right) u_{x}+\left(\beta_{12}^{1}-\beta_{12}^{-1}\right) v_{x}+c_{11} u+c_{12} v\right], \\
u_{x}+\beta v=h\left[\left(\beta_{21}^{1}-\beta_{21}^{-1}\right) u_{x}+c_{21} u+c_{22} v\right] .
\end{array}\right.
$$

Then, choosing $c_{11}=c_{21}=0$ and setting $\gamma_{i j}=\beta_{i j}^{1}-\beta_{i j}^{-1}, i, j=1,2$, from the second equation we have

$$
v_{x}=\frac{1-h \gamma_{21}}{-\beta+h c_{22}} u_{x x}
$$

Replacing this expression in the first equation and neglecting second order terms, we get

$$
u_{t}+\left[\alpha-h\left(\gamma_{11}-c_{12} \frac{1-h \gamma_{21}}{\beta-h c_{22}}\right)\right] u_{x}=\left[\frac{1-h \gamma_{21}}{\beta-h c_{22}}\left(1-h \gamma_{12}\right)+h \frac{q}{2}\right] u_{x x}+O\left(h^{2}+\Delta t\right),
$$


We now look for coefficients $c$, and $\gamma$. such that the drift and the diffusion terms are equal to $\alpha$ and $1 / \beta$ respectively.

- studying the drift term, we have

$$
\tilde{\alpha}=\alpha+h\left(c_{12} \frac{1-h \gamma_{21}}{\beta-h c_{22}}-\gamma_{11}\right)=\alpha+h \frac{c_{12}\left(1-h \gamma_{21}\right)-\gamma_{11}\left(\beta-h c_{22}\right)}{\beta-h c_{22}}
$$

Then, to have $c$, and $\gamma$. independent of $h$, we choose

$$
c_{12}=\gamma_{11} \beta, \quad c_{22}=\gamma_{21} \beta .
$$

- studying the diffusion term, we have

$$
\tilde{\beta}=\frac{1-h \gamma_{21}}{\beta-h c_{22}}\left(1-h \gamma_{12}\right)+h \frac{q}{2}=\frac{2\left(1-h \gamma_{21}\right)\left(1-h \gamma_{12}\right)+h q\left(\beta-h c_{22}\right)}{2\left(\beta-h c_{22}\right)} .
$$

We then write,

$$
\tilde{\beta}=\frac{1}{\beta}+\hat{\beta}
$$

therefore,

$$
\hat{\beta}=\frac{h\left(-2 \beta\left(\gamma_{12}+\gamma_{21}\right)+2 c_{22}+\beta^{2} q\right)+h^{2}\left(2 \beta \gamma_{21} \gamma_{12}-\beta q c_{22}\right)}{2 \beta\left(\beta-h c_{22}\right)} .
$$

For $c_{22}=\gamma_{21} \beta$, we obtain that $\hat{\beta}=0$ if

$$
\gamma_{12}=\frac{\beta q}{2}
$$

To summarize, a class of finite different schemes of the form $(51)$, consistent of order $O\left(h^{2}+\Delta t\right)$ with respect the asymptotic problem (50) is defined by selecting

$$
c_{11}=c_{21}=0, \quad c_{12}=\gamma_{11} \beta, \quad c_{22}=\gamma_{21} \beta, \quad \gamma_{12}=\frac{\beta q}{2} .
$$

There are left some parameters to define, we then look for the monotonicity properties of the scheme.

Proposition 3.4 (Monotonicity). The scheme (51), coupled with (60) and, for $q=\lambda$, with

$$
\tilde{\beta}_{11}^{0}=-\frac{\beta}{2} a_{+}\left(\frac{\lambda^{2}+1}{\lambda}\right), \quad \tilde{\beta}_{22}^{0}=-\frac{\beta}{2} a_{-}\left(\frac{\lambda^{2}+1}{\lambda}\right),
$$

where $a_{ \pm}=\lambda \pm \alpha$, satisfies the monotonicity assumptions (25 - 27) under the conditions:

$$
\begin{gathered}
\tilde{\beta}_{i j}-\left|\tilde{\gamma}_{i j}\right|>\tilde{\beta}_{i j}^{0} \geq 0, \text { for } i \neq j, \\
h<\frac{4 \lambda}{\beta q}, \quad \Delta t \leq \frac{2 \lambda h}{2 \lambda^{2}+h \beta(\lambda+\alpha)\left(\lambda^{2}+1\right)} .
\end{gathered}
$$

To end, from (62), we select

$$
\tilde{\beta}_{i j}^{0}=\frac{1}{2}\left(\tilde{\beta}_{i j}-\left|\tilde{\gamma}_{i j}\right|\right), \text { for } i \neq j
$$


Therefore, the AHOp-coefficients $\tilde{\mathcal{B}}_{-1}, \tilde{\mathcal{B}}_{0}, \tilde{\mathcal{B}}_{1}$, are defined by

$$
\begin{gathered}
\tilde{\mathcal{B}}_{0}=\frac{\beta}{4 \lambda}\left(\begin{array}{cc}
-2 a_{+}\left(\lambda^{2}+1\right) & a_{-} \\
a_{+} & -2 a_{-}\left(\lambda^{2}+1\right)
\end{array}\right), \\
\tilde{\mathcal{B}}_{ \pm 1}=\frac{1}{2}\left(\tilde{B}-\tilde{\mathcal{B}}_{0} \pm \tilde{\Gamma}+h \tilde{C}\right), \\
\tilde{\Gamma}=\frac{\beta q}{2}\left(\begin{array}{cc}
-a_{+} & 0 \\
0 & a_{-}
\end{array}\right), \quad \tilde{C}=\frac{\beta^{2} q}{4 \lambda}\left(\begin{array}{cc}
a_{+} & -a_{-} \\
-a_{+} & a_{-},
\end{array}\right) .
\end{gathered}
$$

\section{NUMERICAL TESTS}

Now, our aim is to show how, for large time simulations, AHO schemes give better numerical results than standard approximations. Therefore, we shall focus our attention on the numerical error as a function of time: for all tests, we shall fix the grid steps $h$ and $\Delta t$ and we will plot the error as the time $t=n \Delta t$ increases. The reference solution is obtained by the Roe-type scheme AHO2-ROE (48), with $h=O\left(10^{-3}\right)$.

\subsection{Test n. 1}

Let us consider system (1), around the stationary and time-asymptotic stable solutions given by

$$
u=u_{0}-b v_{0} e^{p x} / a, \quad v=v_{0} e^{p x},
$$

for some constants $u_{0}, v_{0}$ and for $p=-a d /\left(a c-b^{2}\right)$.

First, we shall compare AHO4 scheme, with the standard first-order pointwise upwind scheme (47), that is actually just an AHO1-UP scheme, and with the AHO2-ROE scheme (48). The parameters in the test are $a=1, c=-a, b=1$ and $d=5$. In Figure 1, we plot the $l^{\infty}$-error for problem as a function of time. Remark that, even if the total time of simulation is $T=5$, this interval is divided in 8 sub-intervals to plot the error curve.

In figures (a) and (b) we have the evolution of the error, when the initial data are taken to be just the stationary solutions. In this case all the schemes do not exactly preserve this stable state, but anyway AHO schemes show a better accuracy, which is proportional to their formal asymptotic order. In figures (c) and (d) we show the evolution of the error when the initial data are given by small compactly supported perturbations of the stationary solutions. In that case, the errors evolve in time, and the AHO2-ROE and the AHO4 improve clearly their accuracy for large times.

\subsection{Test n. 2}

Let us consider system (1), around the constant equilibrium state $u=1$ and $v=0$. The parameters in the test are again $a=1, c=-a, b=1$ and $d=5$. We consider a small compactly supported perturbation of this solution as initial data and we expect a diffusive behavior of the solution, near the corresponding solution of problem (50). Actually what we look for is always a better approximation of the hyperbolic problem, but to obtain that, we need to force the high-order consistency with the main terms in the asymptotic behavior, which are given by the diffusive expansion.

More specifically, here we compare the AHOp scheme (64), with the standard first-order pointwise upwind scheme (47), and with the AHO2-ROE scheme (48). The reference solution is always obtained by the AHO2ROE scheme with $h=O\left(10^{-3}\right)$.

In Figures 2-(a) and 2-(b), we plot the different approximations of the function $u$ at times $T=43$ and $T=350$. In Figures 2-(c) and 2-(d), we plot respectively the $l^{1}$ and $l^{\infty}$ errors as a function of time. Again, even if the total time of simulation is $T=350$, this interval is divided in 8 sub-intervals to plot the error curve.

The numerical results show a clear better performance of the AHOp-scheme in particular for large times, as expected by our asymptotic analysis. 

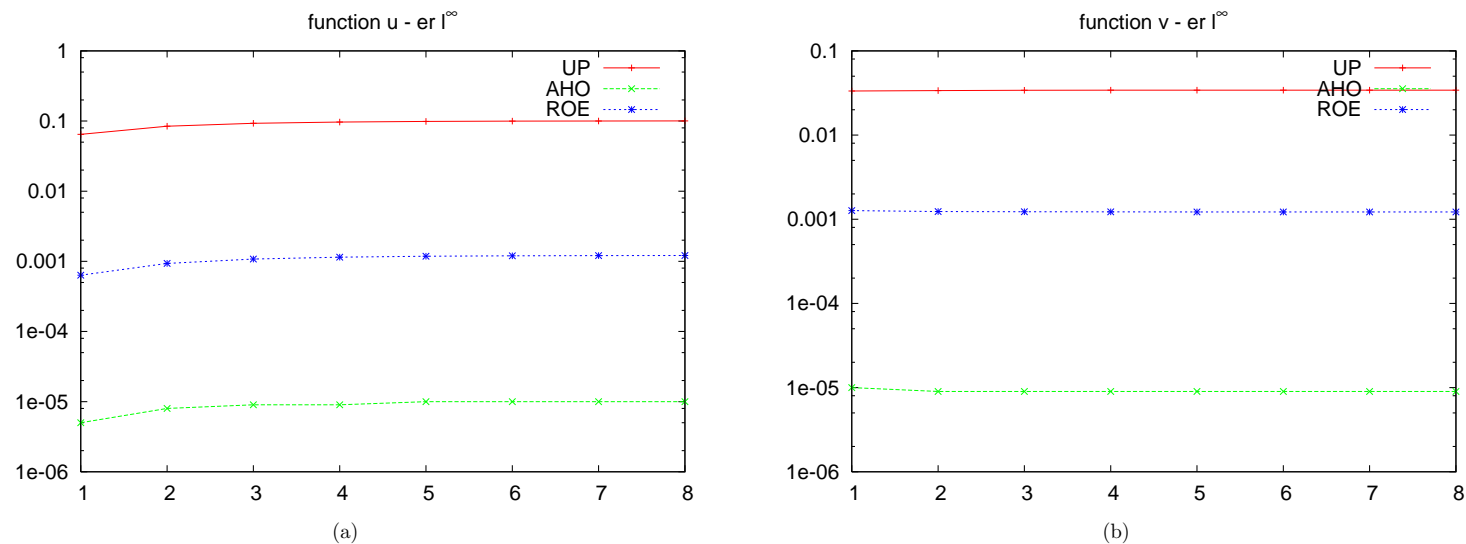

(a)

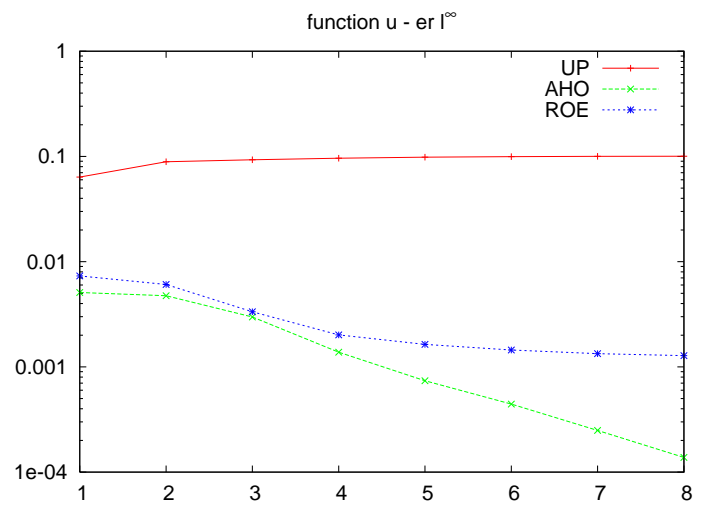

(c)

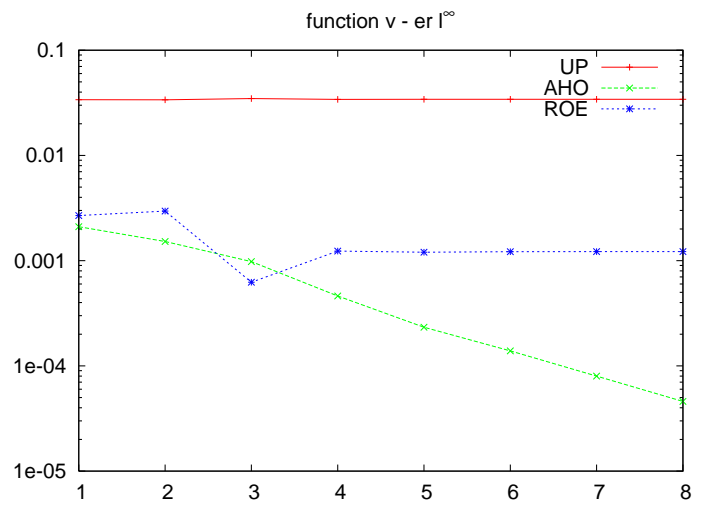

(d)

Figure 1. Test n. 1. (a)-(b) evolution of the $l^{\infty}$-error, when the initial data are taken to be the stationary solutions (65). (c)-(d) evolution of the error, when the initial data are given by a small compactly supported perturbations of the stationary solutions.

\section{ACKNOWLEDGMENTS}

The research activity reported in this paper has been partially conducted with the support of the project "Problemi iperbolici non lineari" for the year 2006 of INDAM-GNAMPA.

\section{REFERENCES}

[1] D. Aregba-Driollet, M. Briani, R. Natalini, Asymptotic high-order schemes for 2x2 dissipative hyperbolic systems, IAC Report 116 (12/2006), to appear in SINUM.

[2] S. Bianchini, B. Hanouzet, R. Natalini, Asymptotic behavior of smooth solutions for partially dissipative hyperbolic systems with a convex entropy, Communications Pure Appl. Math. 60 (2007), 1559-1622.

[3] M. Briani, R. Natalini, Asymptotic high-order schemes for integro-differential problems arising in markets with jumps, Commun. Math. Sci. 4 (2006), 81-96.

[4] G.-Q. Chen, C.D. Levermore, and T.-P. Liu, Hyperbolic conservation laws with stiff relaxation terms and entropy, Comm. Pure and Appl. Math. 47 (1994), 787-830.

[5] B. Hanouzet and R. Natalini, Global existence of smooth solutions for partially dissipative hyperbolic systems with a convex entropy, Arch. Ration. Mech. Anal. 169 (2003), no. 2, 89-117.

[6] L. Hsiao and T.-P. Liu, Convergence to nonlinear diffusion waves for solutions of a system of hyperbolic conservation laws with damping, Comm. Math. Physics 143 (1992), 599-605. 

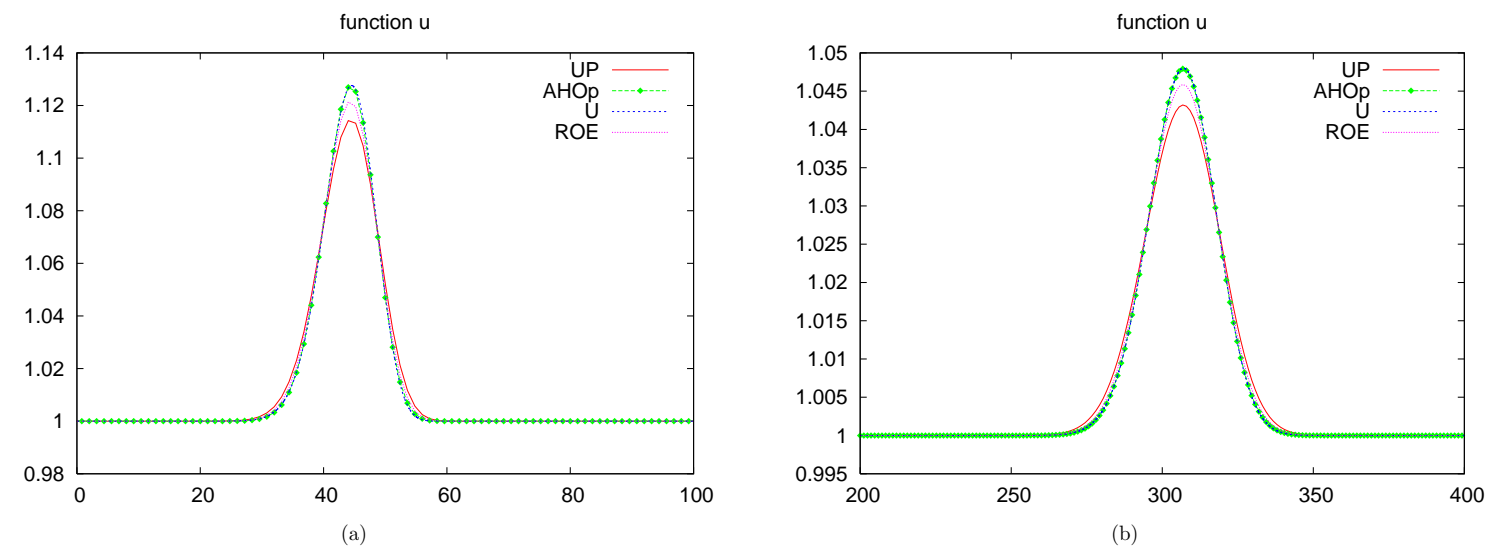

unction $\mathrm{u}$ - error $\mathrm{I}^{1}$
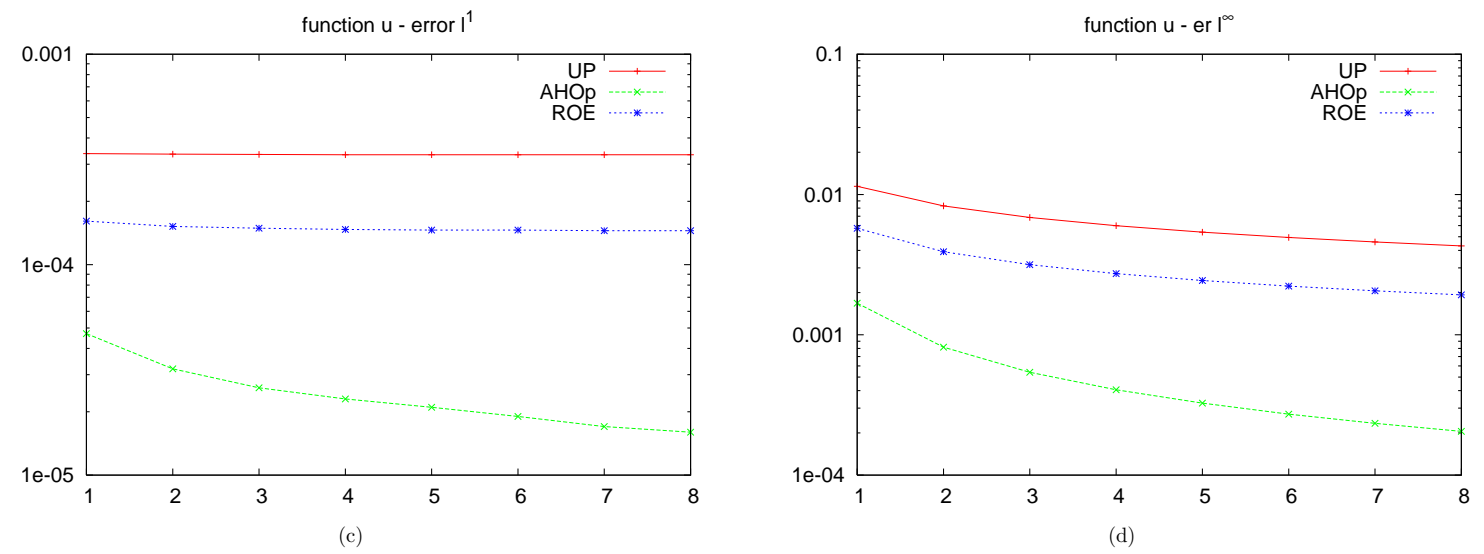

(d)

FiguRE 2. Test n. 2. (a)-(b) different approximations of the function $u$ at times $T=43$ and $T=350$. (c)-(d) evolution of the $l^{1}$ and $l^{\infty}$ errors.

[7] S. Kawashima, Large-time behaviour of solutions to hyperbolic-parabolic systems of conservation laws and applications, Proc. Roy. Soc. Edinburgh Sect. A 106 (1987), no. 1-2, 169-194.

[8] R. Natalini, Recent results on hyperbolic relaxation problems, Analysis of systems of conservation laws (Aachen, 1997), Chapman \& Hall/CRC, Boca Raton, FL, 1999, pp. 128-198.

[9] T. Nishida, Nonlinear hyperbolic equations and related topics in fluid dynamics, Département de Mathématique, Université de Paris-Sud, Orsay, 1978, Publications Mathématiques d'Orsay, No. 78-02.

[10] P.L. Roe. Upwind differencing schemes for hyperbolic conservation laws with source term. Nonlinear Hyperbolic Problems, C.Carasso, P.A.Raviart and D.Serre editors, Lecture Notes in Math., 1270:41-51, 1987.

[11] Y. Shizuta and S. Kawashima, Systems of equations of hyperbolic-parabolic type with applications to the discrete Boltzmann equation, Hokkaido Math. J. 14 (1985), no. 2, 249-275.

[12] T. Sideris, B. Thomases, and D. Wang, Decay and singularities of solutions of the three-dimensional Euler equations with damping, Comm. Partial Differential Equations 28 (2003), no. 3-4, 795-816.

[13] G.B. Whitham, Linear and nonlinear waves, A Wiley \& Sons, New York, 1974. 